\title{
Evolving immunosuppressive microenvironment during human cervical carcinogenesis
}

\author{
A Kobayashi ${ }^{1}$, V Weinberg ${ }^{2}$, T Darragh ${ }^{3}$ and K Smith-McCune ${ }^{1}$
}

\begin{abstract}
Chronic infection with human papillomavirus (HPV) can result in cervical cancer. To understand how HPV escapes immune eradication, we examined biophenotypes of immune cells in human normal cervix, cervical intraepithelial neoplasia (CIN), and cancer. Expression and cellular localization of Forkhead box protein-3 (FOXP3), indolamine 2,3-dioxygenase (IDO), interleukin (IL)-10, and interferon (IFN)- $\gamma$ were examined by immunofluorescence and immunohistochemistry. Mean cell densities of stromal FOXP3 + cells, IDO + cells, IL-10 + cells, CD1a + cells, and macrophages significantly increased from normal cervix to cancer, whereas densities of IFN- $\gamma+$ and MMP-9+ cells increased from normal cervix to CIN but decreased in cancer. Flow cytometry confirmed significant elevation of cervical T cells expressing IFN- $\gamma$ and transforming growth factor- $\beta$ in CIN compared with normal cervix. Upon activation, a significantly increased proportion of cervical T cells expressed IFN- $\gamma$ in CIN than normal. A unique subset of morphologically immature stromal dendritic cells expressing IL-10 and IDO was more numerous in cancer than in normal cervix and CIN. The potentially suppressive immune milieu in the cervix may be permissive of HPV-associated cervical carcinogenesis.
\end{abstract}

\section{INTRODUCTION}

Infection of the cervix by human papillomavirus (HPV) is a common and usually clinically innocuous event. HPV encodes discrete viral proteins that are recognized by the local immune system, with the result that the vast majority of HPV infections are asymptomatic, resolve spontaneously, and result in protective immunity. In rare instances, however, the virus causes dysplastic changes of the cervix known as cervical intraepithelial neoplasia (CIN), which is graded as CIN1, -2 , or -3. CIN lesions are clinically heterogeneous and can spontaneously regress, persist, or in rare instances, progress to invasive cancer. Rates of CIN regression vary with grade, occurring in approximately $50-90 \%$ of women with CIN1, $40 \%$ with CIN2, and $30 \%$ with CIN3. ${ }^{1-3}$ Hence, the immune system can eradicate HPV even in the face of established neoplasia, although the likelihood of clearance declines as the lesions progress along the neoplastic spectrum. The fact that more than 95\% of cervical cancers contain HPV genomes ${ }^{4}$ indicates that in certain cases the immune response is unable to eradicate HPV.

The role of the inflammatory response in HPV infection and disease is multifaceted. On the one hand, clearance of HPV infection involves a cytotoxic immune response. For example, spontaneous regression of HPV-induced genital warts is accom- panied by infiltration of lesions with T cells and macrophages. ${ }^{5}$ Treatment-induced regression using an immune-response modifier, $5 \%$ imiquimod, is accompanied by upregulation of interferon (IFN) $-\alpha,-\beta$, and $-\gamma$, as well as tumor necrosis factor- $\alpha{ }^{6}$ On the other hand, tumor-associated inflammation has been implicated in promoting carcinogenesis, presumably through secretion by inflammatory cells of growth-promoting cytokines, chemokines, angiogenic factors, and proteases. ${ }^{7-12}$ In addition to playing a permissive role, the inflammatory milieu in tumors can actively suppress specific antitumor responses. Polarized macrophages in the tumor microenvironment contribute locally to immunosuppression. ${ }^{9-11}$ A subset of immature or semimature myeloid dendritic cells (DCs) in the tumor microenvironment can produce immunosuppressive cytokines such as interleukin (IL)-10 and transforming growth factor (TGF)- $\beta \cdot{ }^{13-15}$ Immature DCs produce indoleamine 2,3-dioxygenase (IDO), an enzyme that catabolizes tryptophan and thereby suppresses T celldependent immune response. ${ }^{16-18}$ In addition, arginine metabolism by immature DCs contributes to T-cell inhibition by local depletion of arginine, generation of nitric oxide and reactive oxygen species, and other mechanisms. ${ }^{19}$ Regulatory T cells produce T-cell anergy and permit cancer progression through local production of cytokines such as IL-10 and TGF- $\beta .^{20,21} \mathrm{~T}$ cells are

\footnotetext{
${ }^{1}$ Department of Obstetrics, Gynecology, Reproductive Science, University of California at San Francisco, San Francisco, California, USA. ${ }^{2}$ The UCSF Comprehensive Cancer Center Biostatistics Core, University of California at San Francisco, San Francisco, California, USA. ${ }^{3}$ Department of Pathology, University of California at San Francisco, San Francisco, California, USA. Correspondence: K Smith-McCune (kmccune@cc.ucsf.edu)

Received 4 December 2007; accepted 23 April 2008; published online 2 July 2008. doi: 10.1038/mi.2008.33
} 
also inhibited by interaction of the PD-1 receptor with negative costimulatory ligands such as PD-L2 (B7-DC) expressed by DCs and macrophages and PD-L1 (B7-H1) expressed on multiple cell types including antigen-presenting cells. ${ }^{22,23}$ Therefore, there are several mechanisms by which stromal inflammatory cells contribute to tumor escape from immune eradication.

The cervix is an ideal organ for studying the evolution of the stromal microenvironment during carcinogenesis, for several reasons. CIN lesions and cancers are routinely excised for therapy, thereby providing a unique human experimental system for assessing the stromal milieu across the carcinogenic spectrum. CIN and cancer samples express high levels of the HPV oncogene E7, ${ }^{24-26}$ hence cervical cancer represents a dramatic example of failure of the immune response to eliminate an oncogenic pathogen. We have previously shown increased numbers of cells expressing pro-inflammatory (IFN- $\gamma$ ) as well as tolerogenic (IL-10 and TGF- $\beta$ ) factors in CIN2 and/or -3 (high-grade CIN (HGCIN)) compared with normal cervix, suggesting the presence of a dynamic immune equilibrium in precancerous lesions. ${ }^{27}$ In this report, we have characterized the biochemical evolution of the local immune microenvironment in HPV-negative normal cervix, HGCIN, and invasive squamous cancer with regard to the phenotypes of stromal DCs, macrophages, and T cells.

\section{RESULTS}

\section{Demographics}

The mean age of women with samples in the normal cervix group was 58 years (range 43-74), in the HGCIN group was 29 years (range 17-40), and in the cancer group was 43 years (range 26-70). There was a significant overall difference in mean age among the three groups $(P<0.0001)$. Women in the HGCIN group were significantly younger than women with a normal cervix $(P=0.0001)$ or cancer $(P=0.005)$. In addition, women with cancer were significantly younger than those with a normal cervix $(P=0.002)$.

The results of HPV typing showed no HPV detected in any of the eight samples of normal cervix. Several HPV types were detected in HGCIN samples (4 out of 14 samples with HPV16; 2 with HPV 31; 1 each with HPV 18, $-33,-45$, -52 , or -58 ; 1 with an untyped HPV from a probe mix for types $-2,-13,-34,-42$, $-57,-62,-64,-67,-72$, and -82 ; and 2 with undetectable HPV). In the cancer samples, HPV16 was the predominant type found (8 out of 11 samples with HPV16, 1 with HPV18, and 2 with undetectable HPV).

\section{Stromal IFN- $\gamma+$ cells in normal cervix, HGCIN, and cervical cancer}

IFN- $\gamma$ production indicates activation of local cell-mediated immunity, commonly seen in response to viral infection or cancer. We have previously reported that IFN- $\gamma$ was expressed in $\mathrm{CD} 4+$ and $\mathrm{CD} 8+\mathrm{T}$ cells and to a lesser extent in natural killer cells in the cervix, and that the density of IFN- $\gamma+$ cells in the stroma of HGCIN samples was significantly increased compared with normal cervix. ${ }^{27}$ In the current analysis, stroma associated with cervical cancer demonstrated a significant reduction in mean IFN- $\gamma+$ cell density compared with HGCIN (Figure 1a).
The mean age of women contributing samples of HGCIN was significantly lower than that of women contributing samples of normal cervix and cancer. Therefore, we explored whether age and IFN- $\gamma+$ cell density were correlated within each patient subset. No significant correlations existed between age and densities of IFN- $\gamma+$ cells in any of the three groups $(P=1.00$ for normal, $P=0.41$ for $\mathrm{CIN} 2 / 3$, and $P=0.55$ for cancer). Therefore, age is unlikely to contribute to the higher density of IFN $-\gamma+$ cells observed in HGCIN compared with normal or malignant cervix.

\section{DCs in normal cervix, HGCIN, and cancer}

The significant reduction in IFN- $\gamma$ cell density from HGCIN to cervical cancer suggested possible inhibition of IFN- $\gamma$ response during carcinogenesis. As DCs play a key role in directing local immune responses toward either an effector response or tolerance, we analyzed DC biophenotypes in human cervical carcinogenesis. Stromal CD1a + DCs were morphologically immature (lacking pseudopodia) and expressed DC-SIGN (Figure 2a). They also expressed human lymphocyte antigen class II (HLA-II) (Figure 2b), indicating antigen-presenting capability.

Fluorescence-activated cell sorting (FACS) analysis of cells extracted from fresh cervical tissues indicated a higher percentage of CD1a + CD123 + cells expressing DC-SIGN in HGCIN samples than in normal cervix (Figure $\mathbf{1 b}$ and Supplementary Figure 1), suggesting that immature DCs are increased in HGCIN. The majority of cervical CD1a + cells expressed CD123 regardless of disease status (Supplementary Figure 1).

Regarding cytokine production by cervical DCs, we have previously shown that a subset of CD1a + cells in the stroma of HGCIN lesions expressed IL-10. ${ }^{27}$ Our data indicate that immunosuppressive enzyme IDO was expressed by both stromal CD1a + cells (Figure 2b) and IL-10 + cells (data not shown). In normal cervix, stromal CD1a + IDO + cells were widely distributed in the stroma not closely approximated to the epithelium, a distribution pattern similar to CD1a + IL-10 + cells previously reported. ${ }^{27}$ In HGCIN and cervical cancer samples, stromal IDO + cells also localized within lymphocytic infiltrates adjacent to neoplastic epithelium (data not shown). The IDO staining pattern within these cells was cytoplasmic and granular, consistent with that of a secretory protein.

We quantified the densities of stromal CD1a + cells in samples of normal cervix, HGCIN, and cervical cancer, and compared the mean cell densities among the three groups (Figure 1c). There was a significant overall difference in CD1a + cell densities among the three groups $(P=0.0009)$ and a significant increase in CD1a + cell density in cervical cancer samples compared with normal cervix and HGCIN.

These stromal immature DCs expressing immunosuppressive factors described above are distinct from CD1a + Langerhans cells residing within the epithelium. CD1a + cells within the tumor, but not within the stroma, colocalized with S100, a marker for Langerhans cells (data not shown). The majority of $\mathrm{CD} 1 \mathrm{a}+\mathrm{S} 100+\mathrm{DCs}$ within cancer had extended pseudopodia and did not express HLA-II, IDO, or DC-SIGN. The intensity of CD1a staining by immunohistochemistry (IHC) was greater 
a

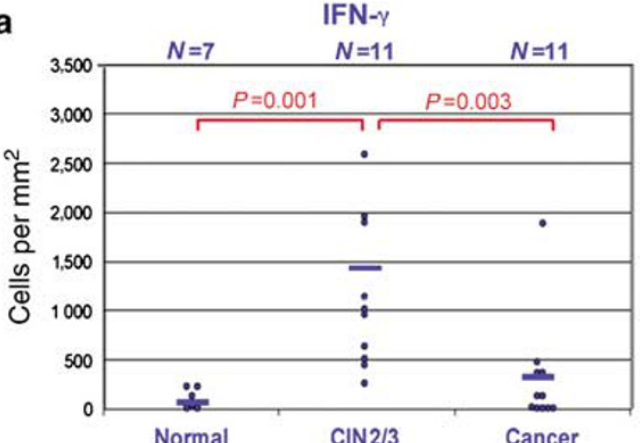

c

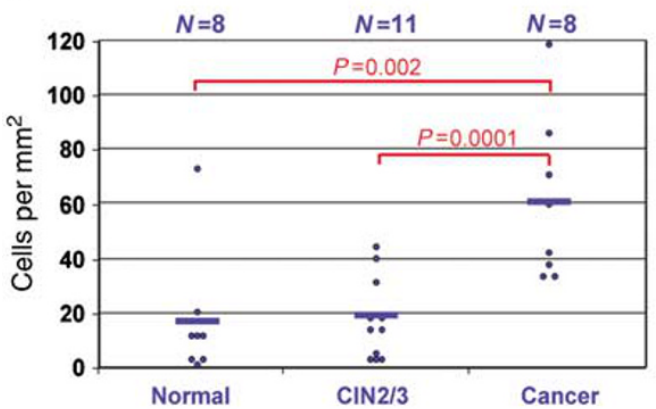

e

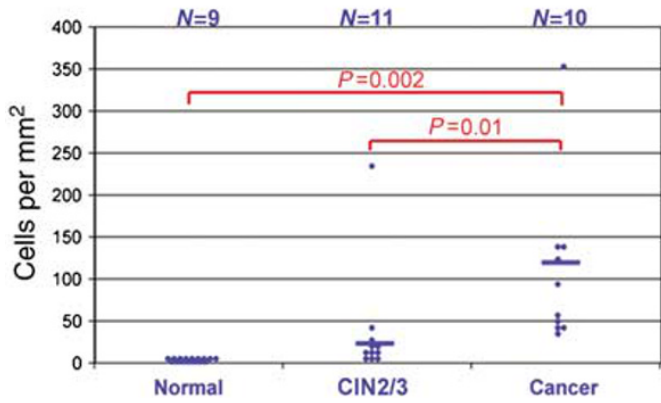

b

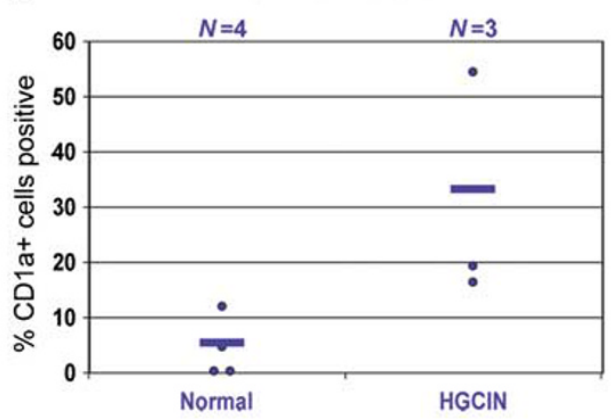

d

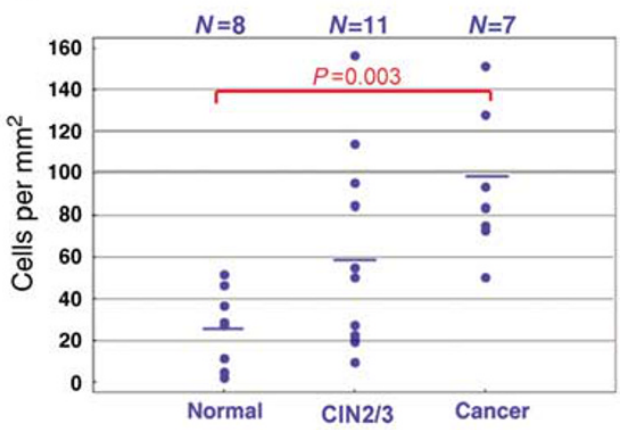

f

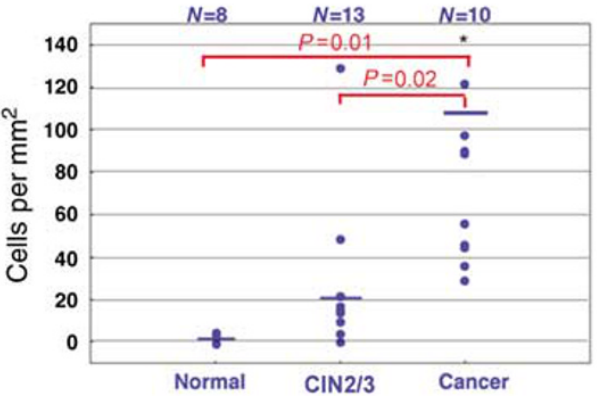

Figure 1 Quantitative comparisons of stromal cell phenotypes in normal cervix, HGCIN, and cancer. (a, c-f) Comparisons of mean cell densities (no. of cells per $\mathrm{mm}^{2}$ ) (MCD) of immunohistochemically stained cells; MCD of each sample is represented by a dot, and the mean of MCDs for each group is represented by a horizontal bar. In $f, M C D$ of one cancer sample was 479 per $\mathrm{mm}^{2}$ and is marked with a black star (not in scale). Statistically significant differences in MCDs are indicated. For each assay, inclusion for analysis was based on availability of appropriate specimens. (b) FACS analysis of CD1a + cells in the cervix in normal and HGCIN samples; $y$ axis is the percentage of CD1a+ cells that are CD123+ and DC-SIGN ${ }^{\text {hi }}$. Each dot represents one sample, and the mean for each group is represented by a horizontal bar. No statistical comparisons were performed due to the small sample size. HGCIN, high-grade CIN.

on cells within tumors than in stroma, and this was confirmed by measuring the mean fluorescence intensity of CD1a staining within tumor versus stroma from one representative sample. The mean fluorescence intensities of nine CD1a + cells within the tumor (range 117-203, median $=165$ ) were significantly higher than the mean fluorescence intensities of $13 \mathrm{CD} 1 \mathrm{a}+$ cells in the stroma (range 44-188, median $=84, P=0.002$ ).

\section{Stromal IL-10 + cells and IDO + cells in normal cervix, HGCIN, and cancer}

We examined stromal alterations in immune-modulating factors during carcinogenesis by quantifying the densities of IL-10+ cells and IDO + cells in normal cervix, HGCIN, and cancer. Among the three groups, there was a significant overall differ- ence among the mean cell densities for both stromal IL-10+ $(P=0.008)$ and IDO $+(P=0.002)$ cells. Pair-wise comparisons revealed significant increases in mean IL-10 + cell density in cervical cancer samples compared with normal cervix samples and in mean IDO + cell density in cervical cancer samples compared with normal cervix or HGCIN samples (Figure 1d and e). Significant trends in increasing density of IDO + and IL-10 + cells were observed in HGCIN lesions compared with normal cervix (Cuzick trend test: $P=0.01$ for each test).

\section{Macrophages in normal cervix, HGCIN, and cancer}

Using CD68 as a marker for macrophages, we quantified the densities of stromal macrophages in samples of normal cervix, HGCIN, and cervical cancer, and compared the mean cell density 
a

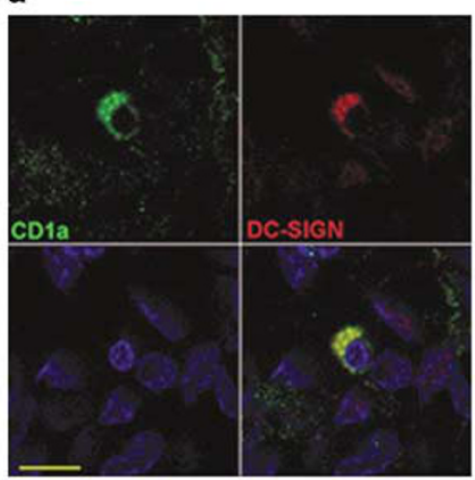

d

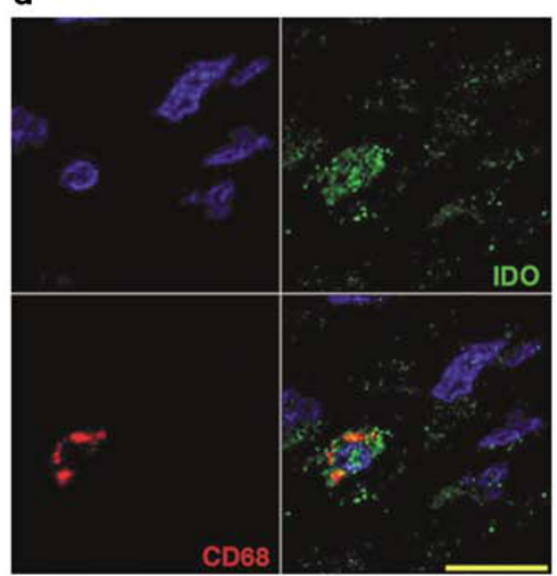

f

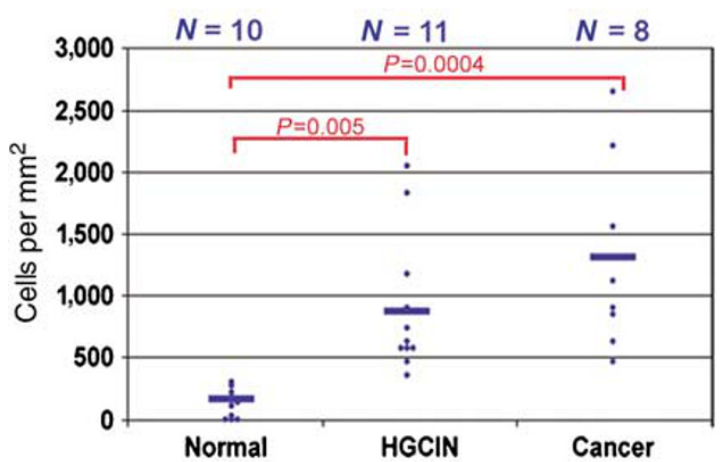

b

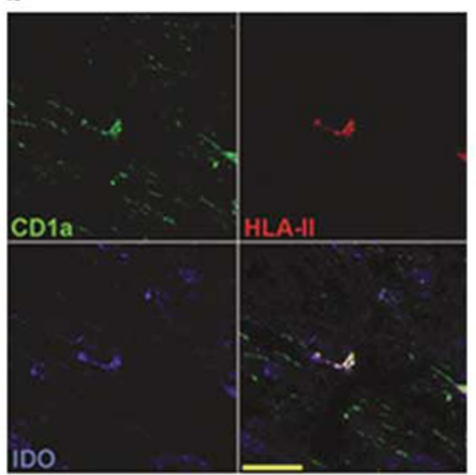

c

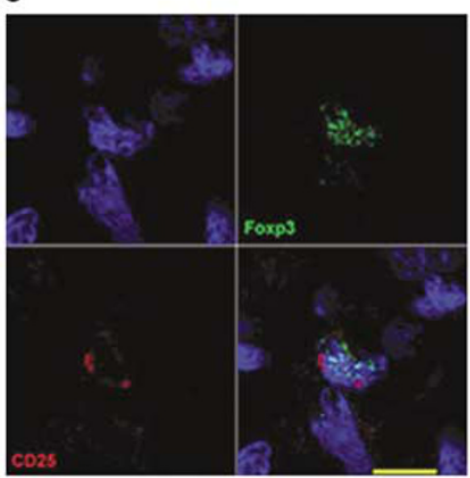

e

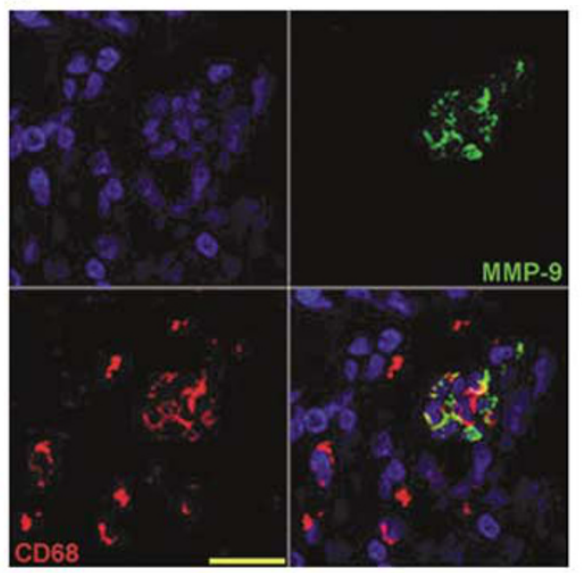

g

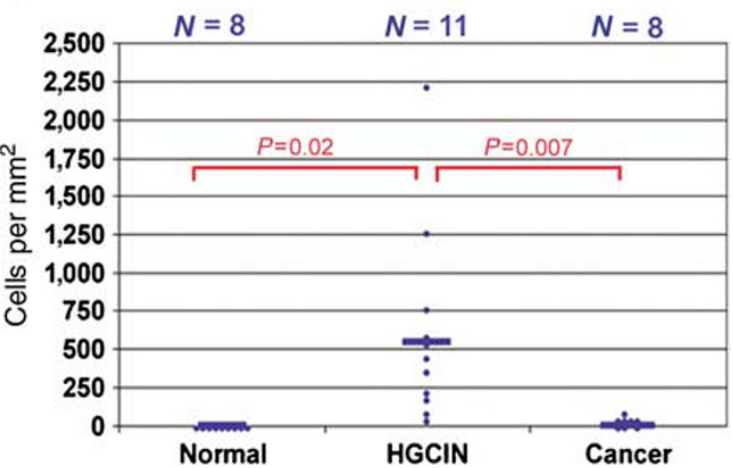

Figure 2 Phenotypic characterization of cervical stromal DCs, T cells, and macrophages, and quantitative comparisons of macrophages in normal cervix, HGCIN, and cancer. (a) Fluorescent double staining of stromal DCs in cervical cancer; CD1a in green, DC-SIGN in red, and DAPI in blue. Bar $=20 \mu \mathrm{m}$. (b) Fluorescent triple staining of stromal DCs in cervical cancer; CD1a in green, HLA-II in red, and IDO in blue. Bar $=20 \mu \mathrm{m}$.

(c) Fluorescent double staining of stromal lymphocytes in cervical cancer: CD25 in red, FOXP3 in green, and DAPI in blue. Bar $=10 \mu \mathrm{m}$. (d and e) Fluorescent double staining of stromal macrophages; CD68 in red, IDO (d) or MMP-9 (e) in green, DAPI in blue. Bar=20 $\mu \mathrm{m}$. (a-e) Right lower images show colocalization of fluorescent staining. ( $\mathbf{f}$ and $\mathbf{g}$ ) Comparisons of MCDs of immunohistochemically stained cells, macrophages (f), and MMP-9+ cells ( $\mathbf{g}$ ) in normal cervix, HGCIN, and cancer. MCD for each sample is represented by a dot, and the mean of MCDs for each group is represented by a horizontal bar. Statistically significant differences in MCDs are indicated. Inclusion for analysis was based on availability of appropriate specimens. DAPI, 4'-6-Diamidino-2-phenylindole; DCs, dendritic cells; HGCIN, high-grade CIN; HLA-II, human lymphocyte antigen class II; IDO, indolamine 2,3-dioxygenase; MMP-9, matrix metallopeptidase-9.

among the three groups (Figure 2f). There was a significant increase in macrophage density in HGCIN and cancer compared with normal cervix. Stromal macrophages in HGCIN and cancer expressed IDO and matrix metallopeptidase (MMP)-9 (Figure 2d and e). Although
MMP-9+ cells were virtually absent in normal cervical stroma, there was a significant increase in the mean cell density of MMP-9+ cells in the stroma of HGCIN, and the density significantly decreased in cancer compared with HGCIN (Figure 2g). 
IDO expression by other cell types in HGCIN and cancer

In tumor-associated stroma, a portion of CD56 + CD3-cells express IDO (data not shown), supporting a previous report that natural killer cells express IDO. ${ }^{28}$ Although T cells and B cells have been reported to express IDO, ${ }^{28}$ neither stromal T cells $(\mathrm{CD} 4+, \mathrm{CD} 8+$, and $\mathrm{CD} 3+)$ nor B cells $(\mathrm{CD} 20+)$ colocalized with IDO in the cervix (data not shown).

IDO staining within the tumor did not colocalize with CD8 or CD3, markers for tumor-infiltrating lymphocytes. However, fluorescent double staining of IDO demonstrated colocalization with cytokeratin 7, a marker for epithelial cancer, indicating that the cancer cells themselves are also a source of IDO (data not shown). The IDO staining pattern in cancer cells was different from that in DCs, natural killer cells, and macrophages, in that it was not confined to the cytoplasm but included nucleis of the tumor cells, consistent with a previous report of IDO staining within cervical cancer. ${ }^{29}$

\section{Putative regulatory T cells in normal cervix, HGCIN, and cancer}

We have previously reported the presence of CD $4+$ CD25+ putative regulatory $\mathrm{T}$ cells in stroma associated with HGCIN lesions. ${ }^{27}$ We further characterized the phenotype of CD4+ T cells in cervical cancer and HGCIN by examining expression of Forkhead box protein-3 (FOXP3), a transcription factor, the expression of which is generally restricted to regulatory $\mathrm{T}$ cells and which is involved in T cell-dependent immune suppression. IHC showed that most FOXP3 + cells were distributed in the stroma of cervical tissues within lymphocytic infiltrates approximated to the neoplastic epithelium, and the vast majority of FOXP3 + cells colocalized with CD3 + (data not shown) and CD25 + cells (Figure 2c). FOXP3 staining localized to nuclei, consistent with its function as a transcription factor. Some FOXP3 + T cells were also observed within cervical cancer (data not shown). It has been shown that some FOXP3 $+\mathrm{T}$ cells can express IFN- $\gamma$ in response to antigenic stimulation. ${ }^{30}$ Triplecolor staining demonstrated that FOXP3 + CD25 + T cells in the cervix did not express IFN- $\gamma$ (Supplementary Figure 2).

Comparing the densities of stromal FOXP3 + cells in normal cervix, HGCIN, and cancer, there was a significant overall difference $(P=0.01)$, and pair-wise comparisons revealed a significant elevation of FOXP3 + cell density in cervical cancer samples compared with normal cervix or HGCIN (Figure 1f). A significant trend in increasing density of FOXP3 + cells was observed in HGCIN lesions compared with normal cervix (Cuzick trend test: $P=0.01$ ).

\section{Intracellular cytokine flow cytometric analysis of cervical T cells}

To assess functional capability of T cells, we used flow cytometry to measure cytokine production in T cells isolated from fresh cervical tissues with or without activation by phorbol 12-myristate 13-acetate and ionomycin. The percentages of viable cervical T cells expressing IFN- $\gamma$ or TGF- $\beta$ were significantly higher in HGCIN than in normal cervix (Table 1; Supplementary Figure 3). The percentages of IFN $-\gamma+$ T cells
Table 1 Comparison of percentages of viable CD3+ cells expressing intracellular cytokines in normal cervix and HGCIN

\begin{tabular}{llll}
\hline Cytokine & $\begin{array}{c}\text { Normal \% } \\
(\mathbf{\pm s . d .}) \mathbf{N}=\mathbf{8}\end{array}$ & $\begin{array}{c}\text { HGCIN \% } \\
\mathbf{( \pm \mathbf { s } . d . )} \mathbf{N = 9}\end{array}$ & P-value \\
\hline $\begin{array}{l}\text { Unactivated } \\
\text { IFN- } \gamma\end{array}$ & $2.3( \pm 1.5)$ & $8.6( \pm 9.5)$ & 0.03 \\
TGF- $\beta$ & $4.0( \pm 2.9)$ & $10.2( \pm 8.0)$ & 0.03
\end{tabular}

Activated $^{\text {a }}$

$\begin{array}{llll}\text { IFN }-\gamma & 16.0( \pm 12) & 30.4( \pm 12.7) & 0.02 \\ \text { TGF- } \beta & 5.9( \pm 3.5) & 12.3( \pm 7.9) & (0.08)\end{array}$

Abbreviations: IFN- $\gamma$ interferon- $\gamma$. HGCIN high-grade CIN : PMA phorbo 12-myristate 13-acetate; TGF- $\beta$, transforming growth factor- $\beta$.

aTreated with PMA and ionomycin as described in Methods.

increased significantly upon activation in both normal cervix and HGCIN ( $P=0.02$ and 0.01 , respectively). The increase in percentages of TGF- $\beta+$ T cells upon activation was significant in normal cervix $(P=0.02)$ but not in $\operatorname{HGCIN}(P=0.14)$. Upon activation, a significantly increased proportion of cervical T cells expressed IFN- $\gamma$ in HGCIN than normal.

When cervical and peripheral blood samples were compared from the same patient, significantly higher proportions of $T$ cells in cervical tissue than in peripheral blood expressed IFN- $\gamma(P=0.01$ for both normal and HGCIN $)$ and TGF- $\beta$ $(P=0.03$ for both normal and HGCIN) (data not shown).

We examined cytokine expression in cervical $\mathrm{T}$ cells from three samples containing histologically confirmed low-grade CIN. In unactivated samples, percentages of cytokine-expressing viable $\mathrm{T}$ cells were low for both $\operatorname{IFN}-\gamma(2.3,2.2$, and $1.4 \%)$ and TGF- $\beta(4.5,1.7$, and $1.3 \%)$, resembling mean values seen in T cells from normal cervix (Table $\mathbf{1}$ ).

\section{DISCUSSION \\ Evolution of DC and macrophage phenotypes during cervical carcinogenesis}

The tumor microenvironment is known to contain immature myeloid cells recruited to the primary tumor site. ${ }^{31}$ In the presence of IL-10 and/or TGF- $\beta$, DCs undergo incomplete differentiation and thereby direct the differentiation of CD4 + T cells into regulatory T cells. ${ }^{15,32-34}$ The stroma of HGCIN lesions is known to contain a novel subset of stromal DCs expressing immunosuppressive factors. ${ }^{27,35}$ The data reported herein demonstrate that stromal DCs in the cervix evolve during carcinogenesis, with progressively increased numbers of cells expressing DC-SIGN and the immunosuppressive factors IL-10 and IDO. This unique subset of stromal CD1a + DCs is distinct from Langerhans cells by virtue of being S100-negative, expressing HLA-II, and expressing lower levels of CD1a. In Figure 3, we propose a model of cervical carcinogenesis in which these novel immature stromal DCs direct the differentiation of regulatory $\mathrm{T}$ cells and thereby determine the polarity of the local immune response.

Our findings that the number of stromal macrophages is increased in HGCIN and cancer confirm those of others, ${ }^{36-39}$ 

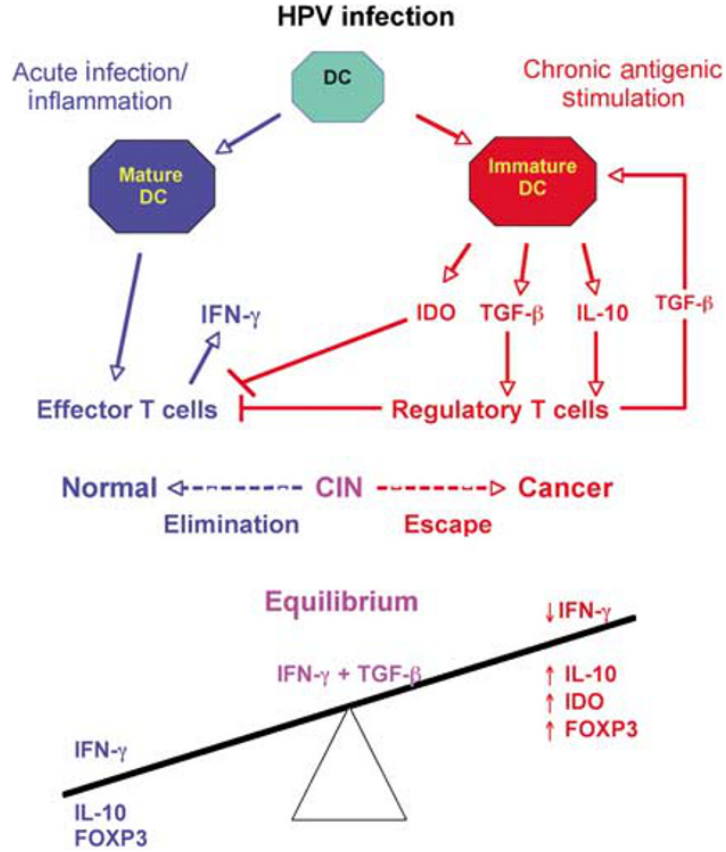

Figure $3 \mathrm{~A}$ model for the central role of DCs in immune editing in HPVinduced cervical disease and the location of HGCIN lesions at the equilibrium between immune escale and immune eradication. Dashed lines are based on our hypotheses. See Discussion for details. DCs, dendritic cells; HGCIN, high-grade CIN; HPV, human papillomavirus.

but we further demonstrate that they have a polarized or alternatively activated phenotype, by virtue of expression of IDO and MMP-9. Their phenotype evolves across the carcinogenic spectrum, given the increase in IDO + but dramatic reduction in MMP-9 + cells in cancer compared with HGCIN lesions. In a murine model of cervical carcinogenesis, MMP-9 produced by infiltrating macrophages has been implicated in angiogenesis and tumor development; genetic or pharmacologic ablation of MMP-9 resulted in diminished bioavailability of vascular endothelial growth factor, reduced microvessel count, diminished progression of CIN lesions to invasive cancer, and smaller tumor volume. ${ }^{40}$ In analogy with data from the transgenic mouse model, increased numbers of MMP-9 + macrophages in human HGCIN may facilitate the pronounced angiogenesis associated with these lesions. ${ }^{41}$

\section{Evolution of T-cell phenotypes during cervical carcinogenesis}

Previous studies have demonstrated that patients with cervical cancer exhibit systemic immune tolerance to HPV antigens. For example, vaccination of cervical cancer patients with HPV antigens failed to generate a cytotoxic T-cell response. ${ }^{42}$ The findings that $\mathrm{T}$ cells in HGCIN lesions expressed increased levels of regulatory cytokines ${ }^{35,43,44}$ and that FOXP3 + T-cell density increased significantly across the disease spectrum to cancer ${ }^{29}$ support a model in which there is a progressive evolution of $\mathrm{T}$-cell phenotypes along the disease spectrum. These data indicate that mechanisms for immune tolerance occur before invasive cancer develops, consistent with the model in Figure 3.
The data presented herein demonstrate for the first time that IDO + cells, FOXP $3+$ T cells, and TGF- $\beta+$ T cells increased across the disease spectrum in parallel with a sharp decline in IFN- $\gamma+$ cells in invasive cancer, suggesting that immunosuppressive mechanisms may dominate in cervical cancer. Differences between peripheral blood and cervix in the proportions of T cells expressing both IFN- $\gamma$ and TGF- $\beta$ reported herein underscore the importance of studying these mechanisms at the mucosal site itself. In a cross-sectional study of invasive cervical cancer samples, increased presence of FOXP3 + cells in lymph nodes was associated with lymph node metastasis, ${ }^{29}$ indicating that immune suppression may extend to sites beyond the tumor itself.

These data support a model of immune equilibrium proposed by others to explain immunologic failure in the emergence of cancer. ${ }^{45,46}$ The finding that low-grade CIN lesions have low numbers of cells expressing FOXP $3,{ }^{29}$ IDO, ${ }^{29}$ and TGF- $\beta$ (data herein) indicates that immunosuppressive mechanisms emerge in HGCIN lesions. We propose that HGCIN lesions are poised at the tipping point between immune eradication and immune escape, as shown in Figure 3, and that the ultimate clinical outcome of HPV-associated disease may depend upon dynamic elements within the stromal microenvironment. Given the cross-sectional nature of our study design, we were unable to test this hypothesis by making correlations with clinical outcomes. In a recent prospective study, however, clearance of HPV infection and low-grade CIN lesions was positively associated with the presence of IFN- $\gamma$ in the cervix, ${ }^{47}$ supporting our model. Appreciation of factors influencing the equilibrium between immune eradication and escape may lead to therapeutics to block immune tolerance and hence accelerate clearance of HGCIN lesions.

\section{METHODS}

Clinical specimens. All specimens of normal cervix, HGCIN, and cervical cancer were obtained as paraffin-embedded blocks from the archives of the Department of Pathology at University of California, San Francisco (UCSF). Normal cervical specimens were obtained from hysterectomies for benign uterine disease with no cervical abnormalities $(N=8)$. HGCIN samples were obtained from loop and cone biopsies performed for standard indications $(N=14)$. Cervical cancer samples were obtained from women undergoing radical hysterectomy for invasive squamous cancer of the cervix $(N=11)$. The histological diagnosis of CIN2 or -3 or squamous cervical cancer was confirmed by T. Darragh. For each assay, inclusion for analysis was based on availability of appropriate specimens.

HPV genotyping. All samples were tested for HPV genotyping using the same paraffin-embedded tissue blocks used in IHC. HPV genotyping was performed on DNA extracted from a $20-\mu \mathrm{m}$ section by PCR using MY09/MY11 primer pairs followed by dot blot hybridization in the laboratory of Joel Palefsky. ${ }^{48}$ Sample adequacy was tested by PCR amplification using specific primers for human $\beta$-globin.

Immunohistochemistry and immunofluorescence. Immunohistochemistry was performed with primary anti-human antibodies against myeloid-derived DCs with anti-CD1a mouse monoclonal antibody (clone 010; DAKO, Carpenteria, CA); against IDO with rabbit polyclonal (generous gift from J.M. McCune at UCSF); against IFN- $\gamma$ and IL-10 (goat polyclonal; R\&D Systems, Minneapolis, MN); 
against CD68 (mouse clone KP1; DAKO); against MMP-9 (rabbit polyclonal; Chemicon International, Temecula, CA); and against FOXP3 (rabbit polyclonal; Abcam, Cambridge, MA). Each primary antibody was used at the following dilutions: 1:100 (CD1a), 1:1500 (IDO), 1:25 (IFN- $\gamma$ ), 1:20 (IL-10), 1:500 (CD68), 1:750 (MMP-9), 1:1500 (FOXP3, lot 61809), and 1:100 (FOXP3, lot 89864). Antigen retrieval for IDO, IL-10, and FOXP3 was performed by digestion with $0.025 \%$ trypsin for $10 \mathrm{~min}$ at $37^{\circ} \mathrm{C}$, boiling the slides in a $1.25 \mathrm{~kW}$ microwave for $2 \mathrm{~min}$ in $10 \mathrm{mM}$ Sorensen's citric buffer ( $\mathrm{pH} 6.5$ ) and cooling in citric buffer for $30 \mathrm{~min}$. Antigen retrieval for CD1a, MMP-9, and CD25 was performed by boiling in citric buffer as described above. For IFN- $\gamma$ and CD68, antigen retrieval was carried out by leaving slides in steamed $1 \mathrm{mM}$ EDTA ( $\mathrm{pH} 8$ ) for $15 \mathrm{~min}$. Before the inactivation of endogenous peroxidase with $10 \%$ hydrogen peroxide, slides for IFN- $\gamma$ staining were blocked for endogenous biotin according to the manufacturer's guidelines (DAKO). IHC for all other antibodies was carried out as previously described. ${ }^{27}$ For IFN- $\gamma$, slides were incubated at 1:400 dilution of biotinylated anti-goat secondary antibody (Jackson ImmunoResearch, West Grove, PA) for $1 \mathrm{~h}$ at room temperature (RT). Rabbit and goat IgG (Jackson ImmunoResearch) and mouse IgG1 and IgG2a (DAKO) were used as negative isotype controls.

Double- and triple-staining immunofluorescence (IF) was performed to characterize CD1a + cells and IDO- or FOXP3-producing cells. Routine IF was performed as described for IHC. EDTA was used for antigen retrieval for HLA-II antibody. Double-staining IF for characterization of IDO- or FOXP3-producing cells was performed as described below. Primary antibodies against cytokeratin 7 (CK7, mouse IgG1, clone OV-TL 12/30; DAKO, at 1:500), IL-10, CD68 (mouse IgG1, clone KP1; DAKO, at 1:100), CD56 (mouse IgG1, clone 123C3.D5; ID Labs, London, ON, Canada, at 1:10), CD4 (mouse IgG1, clone OPD4; DAKO, at 1:100), CD8 (mouse IgG1, clone C8/144B; DAKO, at 1:400), CD20 (mouse IgG2a, clone L26; DAKO, at 1:300), CD3 (mouse IgG1, clone F7.2.38; Gene Tex, San Antonio, TX, at 1:10), or CD25 (mouse IgG1, clone IL2R.1; ID Labs, at 1:100) were combined with IDO or FOXP3 polyclonal rabbit antibody. For double staining of CD1a with DC-SIGN (mouse IgG2b, clone120507; R\&D Systems, at 1:20) and CD56 with CD3 (rabbit IgG; DAKO, at 1:100), trypsin digestion and EDTA were used for antigen retrieval. For double staining of MMP-9 (Chemicon, at 1:600) with CD68, citric buffer was used. All antibodies were incubated on slides overnight at $4{ }^{\circ} \mathrm{C}$ and incubated with corresponding secondary antibodies, Alexa Fluor 488 anti-rabbit IgG, Alexa Fluor 555 anti-mouse IgG, Alexa Fluor 546 anti-mouse IgG2b, and Alexa Fluor 488 anti-mouse IgG1 (all from Molecular Probes, Eugene, OR) at 1:1500, 1:1000, 1:800, and 1:200 dilutions respectively, for $1 \mathrm{~h}$ at RT.

For triple staining, antibodies against HLA-II (monoclonal mouse IgG2a, clone 1QU9; Antigenix America, Huntington Sta., NY) at 1:10 and IDO rabbit antibody were combined and incubated overnight at $4^{\circ} \mathrm{C}$ and incubated with corresponding secondary antibodies (Alexa Fluor 546-conjugated anti-mouse IgG2a and Alexa Fluor 633-conjugated anti-rabbit, both at $1: 200$ ) for $1.5 \mathrm{~h}$ at RT. Slides were then incubated overnight at $4{ }^{\circ} \mathrm{C}$ with anti-CDla antibody, and the following day, slides were incubated with secondary anti-mouse IgG1 antibody conjugated with Alexa Fluor 488 (1:1000). For triple staining of interferon- $\gamma$ with FOXP3 and CD25, slides were incubated with goat anti-INF- $\gamma$ overnight at $4{ }^{\circ} \mathrm{C}$. Alexa Fluor 633 anti-goat secondary antibody at 1:200 was added for $1 \mathrm{~h}$ at RT. Following incubation with non-serum protein block, slides were incubated overnight at $4{ }^{\circ} \mathrm{C}$ with rabbit anti-FOXP3 and mouse anti-CD25 antibodies combined. Corresponding secondary antibodies, Alexa Fluor 488 anti-rabbit at 1:500 and Alexa Fluor 555 anti-mouse at 1:200 were combined and incubated for $1 \mathrm{~h}$ at RT. IF detection, confocal microscopy, and mean fluorescence intensity measurements were performed as previously described. ${ }^{27}$

Quantification of immune cell numbers. The densities of IHC-stained cells in cervical tissues were determined using image analysis software as previously published using Openlab 4.0.3 (Improvision, Lexington,
MA) ${ }^{27}$ For quantification of FOXP 3 + cells, cells were counted as positive only if the staining was nuclear. Cell densities from 5 to 10 fields were averaged for each patient.

The number of samples included in the analysis of each assay is indicated in Table 1 unless otherwise stated in the text. These counts reflect the number of available specimens, which in some cases are less than the total subset stated above due to depletion of the sample reservoir over time.

FACS analysis of fresh cervical tissues. A protocol for fresh tissue collection was approved by the Committee on Human Research at UCSF Normal cervical tissues were collected from patients undergoing elective hysterectomies for indications unrelated to cervical dysplasia; the final pathology report was checked to confirm that the cervix was histologically normal. HGCIN cervical tissues were collected from patients undergoing loop electrosurgical excision for treatment of CIN; samples in which the final diagnosis did not show HGCIN were excluded from the study. For CD1a + cell isolation, fresh tissues were aseptically minced into small pieces $\left(<1 \mathrm{~mm}^{3}\right)$ and were digested by incubation in collagenase type-2 (Worthington Biochemical, Lakewood, NJ) at $0.5 \mathrm{mg} \mathrm{ml}^{-1}$ in Hanks buffer with D-glucose and antibiotics at $37^{\circ} \mathrm{C}$ for $30 \mathrm{~min}$ with agitation. EDTA ( $1 \mathrm{mM}, \mathrm{pH} 7)$ was added for the last $5 \mathrm{~min}$, and minced tissues were disrupted by aspiration through a blunt needle. Cells were collected by filtration through a $70 \mu \mathrm{l}$ filter, rinsed in phosphate-buffered saline with $1 \%$ bovine serum albumin, and incubated with an antibody cocktail of CD1a PE (clone VIT6B; Caltag, Burlingame, CA)/DC-SIGN FITC (clone DCN46; BD Bioscience, San Jose, CA)/CD123 APC (clone AC145; Miltenyi Biotec, Auburn, CA) for FACS analysis.

For intracellular cytokine analysis, minced tissue pieces without digestive enzymes were divided into two wells with $5 \mathrm{ml} \mathrm{RPMI-1640} \mathrm{supple-}$ mented with $15 \% \mathrm{Nu}$-Serum (BD Bioscience), penicillin $\left(100 \mathrm{U} \mathrm{ml}^{-1}\right)$, streptomycin $\left(100 \mu \mathrm{ml}^{-1}\right), 25 \mathrm{mM}$ Hepes, and fungizone $\left(2.5 \mu \mathrm{ml}^{-1}\right)$ and incubated over night at $37^{\circ} \mathrm{C}$. One sample was incubated in medium containing Golgistop (BD) at 1:2500 dilution and the other with $10 \mathrm{ng} \mathrm{ml}^{-1}$ phorbol 12-myristate 13 -acetate and $300 \mathrm{ng} \mathrm{ml}^{-1}$ ionomycin in addition to Golgistop. The following morning, $1 \mathrm{mM}$ EDTA was added to each well, and tissues and medium were transferred into $50 \mathrm{ml}$ conical tubes through $70 \mu$ filter (Falcon/BD), centrifuged at 1000 r.p.m. for $10 \mathrm{~min}$, and washed in phosphate-buffered saline with $1 \%$ bovine serum albumin and $1 \mathrm{mM}$ EDTA. Pelleted cells were incubated with PerCP-conjugated anti-CD3 (clone SK7, BD), APC-anti-CD8 (clone SK1, BD) and FITC-dead/live detection (Molecular Probes) on ice for $30 \mathrm{~min}$ in the dark and washed in phosphate-buffered saline with $1 \%$ bovine serum albumin and $1 \mathrm{mM}$ EDTA. Intracellular cytokine staining with PE-anti-IFN- $\gamma$ (clone 25723.11; BD) and PE-anti-TGF- $\beta$ (clone TB21; IQProducts, DL Groningen, The Netherlands) was carried out following the manufacturer's guidelines (Cytofix/Cytoperm Plus Kit; BD Permingen, San Diego, CA). For both CD1a and intracellular cytokine analyses, stained cells were fixed in $4 \%$ paraformaldehyde and stored at $4{ }^{\circ} \mathrm{C}$ till flow cytometry (FACS Calibur; BD Immunocytometry Systems, San Jose, CA). Cytometry data were analyzed with FlowJo software (Tree Star, Ashland, OR).

Statistical analyses. For each antibody, the overall comparison among the three patient groups of mean cell densities was performed using analysis of variance methods recognizing the small sample sizes. When the overall comparison was statistically significant, post hoc pair-wise tests were performed using the Newman-Keuls method to determine where differences occurred. The non-parametric Cuzick test for ordered groups was performed to test for a trend in cell density with increasing disease state. Due to the small number of patients in each subset, the data were also analyzed using the non-parametric Kruskal-Wallis test (results not shown). The comparison was defined as being significantly different only when significance was confirmed. The non-parametric Spearman rank correlation was calculated to evaluate the pair-wise association between variables (e.g., age and IFN- $\gamma$ ). The non-parametric Wilcoxon test for matched pairs was used for the analysis of flow cytometry data 
to compare the distributions of cervical T cells with or without activation for HGCIN and for normal cervix, and to compare the distributions of cervical T cells with peripheral blood T cells for HGCIN and for normal cervix. To evaluate no activation and activation separately, the Mann-Whitney test was used to compare the distributions of normal cervical T cells and HGCIN T cells. Significance was defined as a probability value less than 0.05 . No adjustment for multiple comparisons was performed in these analyses. Probability values are stated in the text.

SUPPLEMENTARY MATERIAL is linked to the online version of the paper at $\mathrm{http} / / / \mathrm{www}$.nature.com/mi

\section{ACKNOWLEDGMENTS}

Supported by a Research Scholars grant from the American Cancer Society (to K.S.M.) and NIH/NCI P30 CA 82103 Cancer Center support grant to V.W. and to K.S.M. (PI Frank McCormick). We thank Jeff Mold and M.J. McCune from UCSF School of Medicine for providing rabbit antibody against IDO.

\section{DISCLOSURE}

The authors declared no conflict of interest.

(c) 2008 Society for Mucosal Immunology

\section{REFERENCES}

1. Melnikow, J., Nuovo, J., Willan, A.R., Chan, B.K. \& Howell, L.P. Natural history of cervical squamous intraepithelial lesions: a meta-analysis. Obstet. Gynecol. 92, 727-735 (1998).

2. Moscicki, A.B. et al. Regression of low-grade squamous intra-epithelial lesions in young women. Lancet 364, 1678-1683 (2004).

3. Ostor, A.G. Natural history of cervical intraepithelial neoplasia: a critical review. Int. J. Gynecol. Pathol. 12, 186-192 (1993).

4. Munoz, N. et al. Epidemiologic classification of human papillomavirus types associated with cervical cancer. N. Engl. J. Med. 348, 518-527 (2003).

5. Coleman, N. et al. Immunological events in regressing genital warts. Am. J. Clin. Pathol. 102, 768-774 (1994).

6. Tyring, S. Immune response modification: imiquimod. Australas. J. Dermatol. 39 (Suppl 1), S11-S13 (1998)

7. Balkwill, F. \& Coussens, L.M. Cancer: an inflammatory link. Nature 431, 405-406 (2004).

8. Lewis, C.E. \& Pollard, J.W. Distinct role of macrophages in different tumor microenvironments. Cancer Res. 66, 605-612 (2006).

9. Pollard, J.W. Tumour-educated macrophages promote tumour progression and metastasis. Nat. Rev. Cancer 4, 71-78 (2004).

10. Mantovani, A., Sica, A. \& Locati, M. Macrophage polarization comes of age. Immunity 23, 344-346 (2005)

11. Mantovani, A., Schioppa, T., Porta, C., Allavena, P. \& Sica, A. Role of tumor-associated macrophages in tumor progression and invasion. Cancer Metastasis Rev. 25, 315-322 (2006).

12. Balkwill, F. Cancer and the chemokine network. Nat. Rev. Cancer 4, 540-550 (2004).

13. Pinzon-Charry, A., Maxwell, T. \& Lopez, J.A. Dendritic cell dysfunction in cancer: a mechanism for immunosuppression. Immunol. Cell Biol. 83, 451-461 (2005).

14. Zou, W. Immunosuppressive networks in the tumour environment and their therapeutic relevance. Nat. Rev. Cancer 5, 263-274 (2005).

15. Wakkach, A. et al. Characterization of dendritic cells that induce tolerance and T regulatory 1 cell differentiation in vivo. Immunity $\mathbf{1 8}$ 605-617 (2003).

16. Uyttenhove, C. et al. Evidence for a tumoral immune resistance mechanism based on tryptophan degradation by indoleamine 2,3-dioxygenase. Nat. Med. 9, 1269-1274 (2003).

17. Hwu, P. et al. Indoleamine 2,3-dioxygenase production by human dendritic cells results in the inhibition of T cell proliferation. J. Immunol. 164, 3596-3599 (2000).

18. Mellor, A.L. et al. Cutting edge: induced indoleamine 2,3 dioxygenase expression in dendritic cell subsets suppresses T cell clonal expansion. J. Immunol. 171, 1652-1655 (2003).

19. Bronte, V. \& Zanovello, P. Regulation of immune responses by L-arginine metabolism. Nat. Rev. Immunol. 5, 641-654 (2005).
20. Sakaguchi, S. et al. Immunologic tolerance maintained by CD25+ CD4+ regulatory $T$ cells: their common role in controlling autoimmunity, tumor immunity, and transplantation tolerance. Immunol. Rev. 182, 18-32 (2001).

21. Shimizu, J., Yamazaki, S. \& Sakaguchi, S. Induction of tumor immunity by removing CD25+CD4+T cells: a common basis between tumor immunity and autoimmunity. J. Immunol. 163, 5211-5218 (1999).

22. Latchman, Y.E. et al. PD-L1-deficient mice show that PD-L1 on T cells, antigen-presenting cells, and host tissues negatively regulates $T$ cells. Proc. Natl. Acad. Sci. USA 101, 10691-10996 (2004).

23. Zhang, Y. et al. Regulation of T cell activation and tolerance by PDL2 Proc. Natl. Acad. Sci. USA 103, 11695-11700 (2006).

24. Daniel, B., Rangarajan, A., Mukherjee, G., Vallikad, E. \& Krishna, S. The link between integration and expression of human papillomavirus type 16 genomes and cellular changes in the evolution of cervical intraepithelial neoplastic lesions. J. Gen. Virol. 78 (Part 5), 1095-1101 (1997).

25. Durst, M., Glitz, D., Schneider, A. \& zur Hausen, H. Human papillomavirus type 16 (HPV 16) gene expression and DNA replication in cervical neoplasia: analysis by in situ hybridization. Virology 189, 132-140 (1992).

26. Stoler, M.H. et al. Human papillomavirus type 16 and 18 gene expression in cervical neoplasias. Hum. Pathol. 23, 117-128 (1992).

27. Kobayashi, A. et al. Functional attributes of mucosal immunity in cervical intraepithelial neoplasia and effects of HIV infection. Cancer Res. 64, 6766-6774 (2004).

28. Kai, S. et al. Indoleamine 2,3-dioxygenase is necessary for cytolytic activity of natural killer cells. Scand. J. Immunol. 59, 177-182 (2004).

29. Nakamura, T. et al. Expression of indoleamine 2, 3-dioxygenase and the recruitment of Foxp3-expressing regulatory $T$ cells in the development and progression of uterine cervical cancer. Cancer Sci. 98, 874-881 (2007).

30. Sawitzki, B. et al. IFN-gamma production by alloantigen-reactive regulatory T cells is important for their regulatory function in vivo. J. Exp. Med. 201, 1925-1935 (2005).

31. Kim, R., Emi, M., Tanabe, K. \& Arihiro, K. Tumor-driven evolution of immunosuppressive networks during malignant progression. Cancer Res. 66, 5527-5536 (2006)

32. Geissmann, F. et al. TGF-beta 1 prevents the noncognate maturation of human dendritic Langerhans cells. J. Immunol. 162, 4567-4575 (1999).

33. Gad, M., Claesson, M.H. \& Pedersen, A.E. Dendritic cells in peripheral tolerance and immunity. APMIS 111, 766-775 (2003).

34. Jonuleit, H., Schmitt, E., Schuler, G., Knop, J. \& Enk, A.H. Induction of interleukin 10-producing, nonproliferating CD4(+) T cells with regulatory properties by repetitive stimulation with allogeneic immature human dendritic cells. J. Exp. Med. 192, 1213-1222 (2000).

35. Giannini, S.L. et al. Cytokine expression in squamous intraepithelial lesions of the uterine cervix: implications for the generation of local immunosuppression. Clin. Exp. Immunol. 113, 183-189 (1998).

36. Hammes, L.S. et al. Macrophages, inflammation and risk of cervical intraepithelial neoplasia (CIN) progression - clinicopathological correlation. Gynecol. Oncol. 105, 157-165 (2007).

37. al-Saleh, W. et al. Inverse modulation of intraepithelial Langerhans' cells and stromal macrophage/dendrocyte populations in human papillomavirus-associated squamous intraepithelial lesions of the cervix. Virchows Arch. 427, 41-48 (1995).

38. Hachisuga, T., Fukuda, K., Hayashi, Y., Iwasaka, T. \& Sugimori, H. Immunohistochemical demonstration of histiocytes in normal ectocervical epithelium and epithelial lesions of the uterine cervix. Gynecol. Oncol. 33, 273-278 (1989).

39. Tay, S.K., Jenkins, D., Maddox, P., Hogg, N. \& Singer, A. Tissue macrophage response in human papillomavirus infection and cervical intraepithelial neoplasia. Br. J. Obstet. Gynaecol. 94, 1094-1097 (1987).

40. Giraudo, E., Inoue, M. \& Hanahan, D. An amino-bisphosphonate targets MMP-9-expressing macrophages and angiogenesis to impair cervical carcinogenesis. J. Clin. Invest. 114, 623-633 (2004).

41. Smith-McCune, K.K. \& Weidner, N. Demonstration and characterization of the angiogenic properties of cervical dysplasia. Cancer Res. 54, 800-804 (1994).

42. Ressing, M.E. et al. Detection of Thelper responses, but not of human papillomavirus-specific cytotoxic T lymphocyte responses, after peptide vaccination of patients with cervical carcinoma. J. Immunother. 23, 255266 (2000). 


\section{ARTICLES}

43. Clerici, M. et al. Cytokine production patterns in cervical intraepithelial neoplasia: association with human papillomavirus infection. J. Natl. Cancer Inst. 89, 245-250 (1997).

44. El-Sherif, A.M., Seth, R., Tighe, P.J. \& Jenkins, D. Quantitative analysis of IL-10 and IFN-gamma mRNA levels in normal cervix and human papillomavirus type 16 associated cervical precancer. J. Pathol. 195, 179-185 (2001).

45. Dunn, G.P., Old, L.J. \& Schreiber, R.D. The immunobiology of cancer immunosurveillance and immunoediting. Immunity 21, 137-148 (2004).
46. Kim, R., Emi, M. \& Tanabe, K. Cancer immunoediting from immune surveillance to immune escape. Immunology 121, 1-14 (2007).

47. Song, S.H. et al. Interferon-gamma (IFN-gamma): a possible prognostic marker for clearance of high-risk human papillomavirus (HPV). Gynecol. Oncol. 108, 543-548 (2008).

48. Palefsky, J.M., Holly, E.A., Ralston, M.L. \& Jay, N. Prevalence and risk factors for human papillomavirus infection of the anal canal in human immunodeficiency virus (HIV)-positive and HIV-negative homosexual men. J. Infect. Dis. 177, 361-367 (1998). 